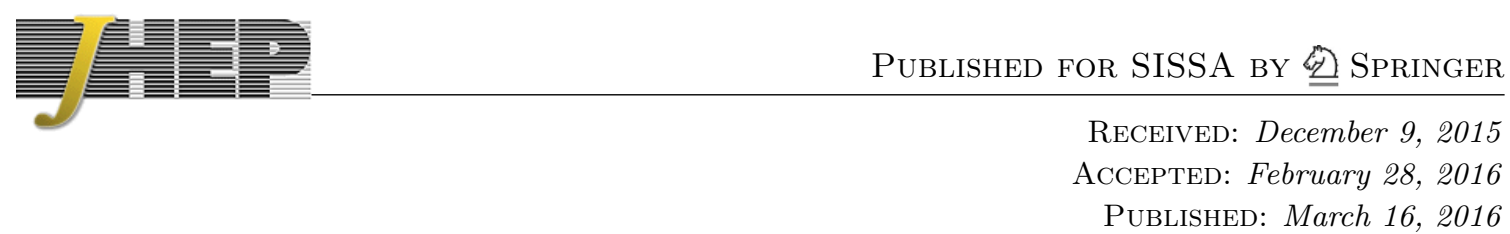

\title{
Primordial gravity wave background anisotropies
}

\author{
Vasyl Alba ${ }^{a}$ and Juan Maldacena ${ }^{b}$ \\ ${ }^{a}$ Department of Physics, Princeton University, \\ Princeton, NJ, U.S.A. \\ ${ }^{b}$ Institute for Advanced Study, \\ Princeton, NJ, U.S.A. \\ E-mail: valba@princeton.edu, malda@ias.edu
}

ABSTRACT: We consider the primordial gravity wave background produced by inflation. We compute the small anisotropy produced by the primordial scalar fluctuations.

KeYwords: Classical Theories of Gravity, Cosmology of Theories beyond the SM

ARXiv EPrint: 1512.01531 


\section{Contents}

1 Introduction 1

2 Gravity waves from inflation at leading order 1

3 Anisotropy 3

\section{Introduction}

The theory of inflation predicts a stochastic background of gravity waves, which were produced by quantum effects [1-10]. To leading order, this is a statistically homogeneous and isotropic background. However, at higher orders we expect some influence from the scalar fluctuations. In the same way that the cosmic microwave radiation is not isotropic, but has small anisotropies of order $10^{-5}$, the gravity wave background is also not isotropic but has small anisotropies of the same order, also due to the scalar fluctuations. At relatively large angles, which correspond to scalar fluctuations entering the horizon during matter domination, the main effect is due to the Sachs-Wolfe effect [11]. Namely we start locally with the same spectrum of gravity waves, which are then red or blue shifted by an amount proportional to the amplitude of the scalar fluctuations. The overall observable effect then depends on the frequency dependence of the spectrum of the gravity waves, which is determined by the properties of the universe when the gravity wave mode crosses the horizon, both exiting and reentering.

Of course, given that we have not yet measured the leading order gravity waves, the effects that we discuss in this paper are not going to be measured in the near future. However, it is a theoretically interesting effect, and we trust in the ingenuity of current and future experimentalists!

This paper is organized as follows. We first give a quick review of the leading order gravity wave background. Then, we discuss its anisotropies, focusing on relatively large angles which correspond to scalar fluctuations that entered the horizon after matter domination. We also focus on gravity waves that entered the horizon in the radiation dominated era, which would correspond to wavelengths that could be measured directly by gravity wave detectors.

\section{Gravity waves from inflation at leading order}

We can view the leading order effect as the result of a Bogoliubov transformation between the vacuum in the very early inflationary period and the vacuum today [1]. In other words, expanding the Einstein action to quadratic order we get an action for each of the two polarization components of the gravity waves. Each polarization component obeys an equation 


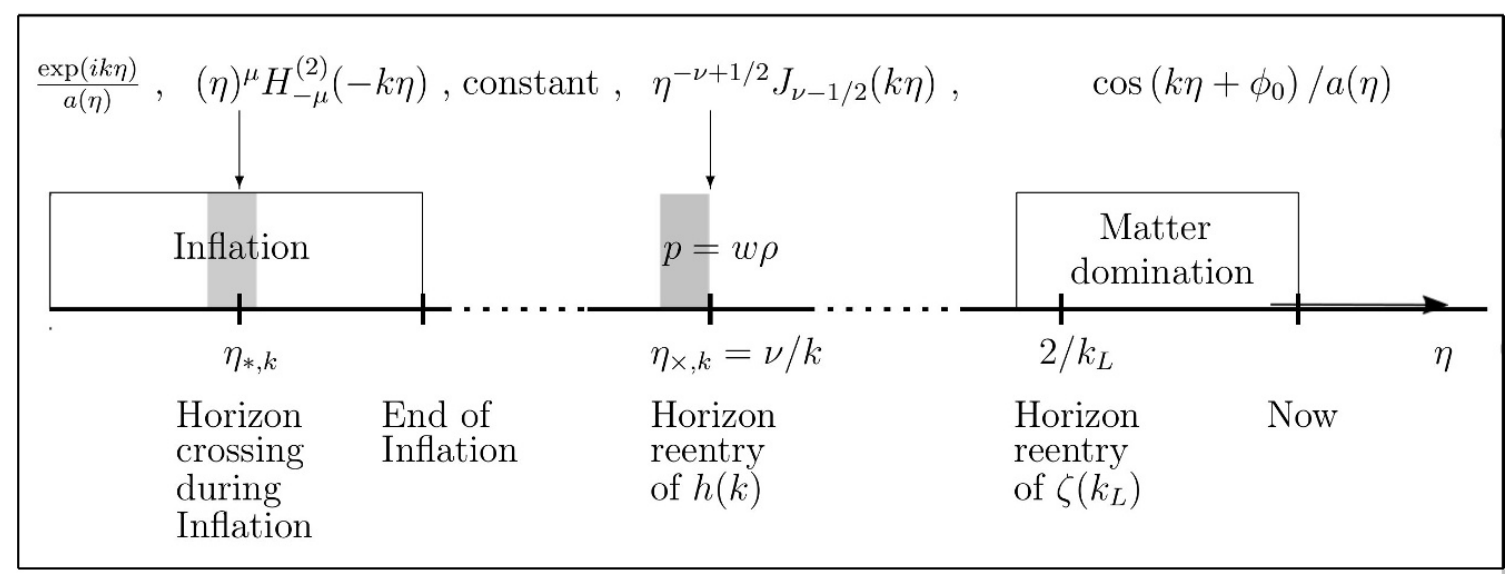

Figure 1. Summary of the evolution of a single mode during the history of the universe. We need to know the equation of state only in the shaded regions. The first shaded region occurs during slow-roll inflation. The second shaded region takes place during an epoch with equation of state $p=w \rho$. Both in the far past and present time the WKB approximation is valid. Outside the horizon the solution has constant amplitude. Long wavelength scalar fluctuation modes entered horizon during the matter dominated era that is assumed to last until now. One the top line we have written the form of the solution in each region.

equal to that of a minimally coupled scalar field in the spatially uniform time dependent cosmological solution. This background metric can be written as $d s^{2}=a(\eta)^{2}\left(-d \eta^{2}+d \vec{x}^{2}\right)$, where $\eta$ is conformal time. Due to translation symmetry, we can focus on one comoving momentum mode $\vec{k}$ at a time. Each of these modes undergoes the following history. It starts its life well inside the horizon in the adiabatic vacuum. It then exits the horizon during inflation at some time $\eta_{*, k}$, where $\eta_{*, k} k=1$. It then remains with constant (time independent) amplitude until it reenters the horizon. When the mode is well inside the horizon it is oscillating and redshifting as any other massless particle. See figure 1. The solution well inside the horizon is given by the WKB approximation. In order to match the solution well inside to the one well outside the horizon, we need to solve the equation during horizon crossing. During horizon crossing we can approximate the evolution of the background in terms of a fluid with a constant $w$, where $p=w \rho$, so that $a(\eta) \propto \eta^{\frac{2}{1+3 w}}$. Then the solution becomes a combination of Bessel functions. When the mode is exiting the horizon during inflation, the appropriate solution is proportional to $\eta^{\mu} H_{-\mu}^{(2)}(-k \eta)$, with $\mu=\frac{3}{2}+\epsilon$, where $\epsilon$ is the standard slow roll parameter $\left(\epsilon=-\dot{H} / H^{2}\right)$ and $k=|\vec{k}|$. Similarly, when the mode reenters the horizon when the universe is dominated by a fluid with an equation of state with fixed $w$, then the solution is proportional to $\eta^{-\nu+\frac{1}{2}} J_{\nu-\frac{1}{2}}(k \eta)$, with $\nu=2 /(1+3 w)$. We will call the time of horizon reentry, $\eta_{\times, k}$, defined by setting $k=a\left(\eta_{\times, k}\right) H\left(\eta_{\times, k}\right)$ or $k \eta_{\times, k}=\nu$.

Matching the solutions we find that the normalized solution has a late time behavior of the form

$$
h(k, \eta)=\frac{H\left(\eta_{*, k_{0}}\right)}{\sqrt{2} k^{\frac{3}{2}} M_{p l}}\left(\frac{k_{0}}{k}\right)^{\epsilon+\nu} \frac{\Gamma\left(\nu+\frac{1}{2}\right)}{\sqrt{\pi}}\left(\frac{2}{\nu}\right)^{\nu} \frac{a\left(\eta_{\times, k_{0}}\right)}{a(\eta)} \cos \left(k \eta+\phi_{0}\right),
$$


Here $k_{0}$ is a reference comoving momentum. ${ }^{1}$ The factor of $\left(\frac{k_{0}}{k}\right)^{\epsilon+\nu}$ arises because, as we move $k$ away from $k_{0}$, we are crossing the horizon earlier or later. Here $\phi_{0}$ is the initial phase of the wave, which is $-\frac{\pi \nu}{2}$, but is unmeasurable for the gravity waves we consider. The redshift after horizon reentry is encoded in the factor $1 / a(\eta)$ in $(2.1)$.

The final density of gravity waves is proportional to the square of (2.1). We can express it in terms of the energy density or number density as follows

$$
\begin{aligned}
d \rho_{g} & =2 \omega \frac{\omega^{2} d \omega}{(2 \pi)^{3}} d \Omega\left\langle N_{\vec{k}}\right\rangle, \quad \text { with } \\
\left\langle N_{\vec{k}}\right\rangle & =\frac{H\left(\eta_{*, k}\right)^{2}}{H\left(\eta_{\times, k}\right)^{2}} C_{\nu}=\frac{H\left(\eta_{*, k_{0}}\right)^{2}}{H\left(\eta_{\times, k_{0}}\right)^{2}}\left(\frac{k_{0}}{k}\right)^{2(\nu+1+\epsilon)} C_{\nu} \\
C_{\nu} & \equiv \frac{\Gamma\left(\nu+\frac{1}{2}\right)^{2}}{4 \pi}\left(\frac{2}{\nu}\right)^{2 \nu}
\end{aligned}
$$

where $\left\langle N_{\vec{k}}\right\rangle$ is a density of states as a function of $k$ and $\omega=k / a$ is the physical energy of the waves. The factor of two comes from the two polarization states. It is useful to think in terms of $N_{k}$ because this is conserved after horizon reentry. Notice that $N_{k}$ is essentially given by the ratio of the Hubble constants at horizon crossing. We have also made more explicit the form of the spectrum by defining an arbitrary reference momentum $k_{0}$. In the case of photons we would get the usual thermal distribution for $N_{k}$. We see that the spectrum is determined by the equation of state at the horizon crossing times. More generally, the spectrum of gravity waves encodes the whole expansion history of the universe [12-16].

\section{Anisotropy}

We are interested in the anisotropy of the gravity waves that comes from the interaction with the scalar fluctuations. We will concentrate on the anisotropy at relatively large angular scales, which is produced by long wavelength scalar fluctuations. More precisely, we consider a scalar fluctuation mode with a momentum $k_{L}$ which reenters the horizon during the matter dominated era. In order to find the effect on the gravity waves, it is important to solve the equation of motion for the mode $\zeta_{k_{L}}$ after it reenters the horizon. We then consider the propagation of the gravity waves through that perturbed universe. This computation is essentially identical to one done for photons, which is the so called Sachs-Wolfe effect [11]. We can work in the gauge where the matter density is constant so that the fluctuation is purely on the scale factor of the spatial part of the metric of the surfaces with constant density. In that gauge $\zeta_{k_{L}}$ continues to be constant after crossing the horizon, but the metric develops a $g_{0 i}$ component that should be computed by first considering $w>0$ and taking $w \rightarrow 0$ at the end of the computation [17]. ${ }^{2}$ Then the perturbed metric has the form $d s^{2}=a^{2}\left[-d \eta^{2}+(1+2 \zeta) d \vec{x} d \vec{x}-\frac{4}{5 a H} \partial_{i} \zeta d \eta d x^{i}\right]$. Solving the geodesic equation on this perturbed metric we find that the extra redshift for a massless

\footnotetext{
${ }^{1}$ In other words, if we are interested in a certain range of values of $k$, we can choose $k_{0}$ to be somewhere in that range. There is no dependence on $k_{0}$ due to the dependence on $\eta_{*, k_{0}}$ and $\eta_{\times, k_{0}}$.

${ }^{2}$ See equations (35)-(38) in [17]. We thank M. Zaldarriaga for sharing some notes on this with us.
} 


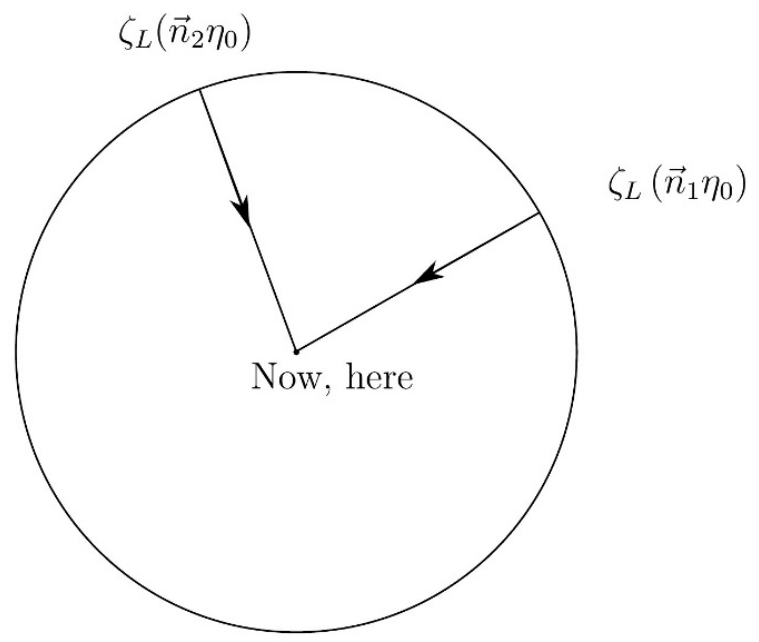

Figure 2. Gravitons that are coming from different directions have different redshift, because values of a scalar perturbation $\zeta_{L}$ are different at different emission points.

particle emitted at an early time and observed today is

$$
\frac{\delta \omega}{\omega}=\frac{1}{5}\left[\zeta_{\text {today }}-\zeta_{\text {emitted }}\right]
$$

where $\zeta_{\text {today }}$ is the value at the location of the observations today, while $\zeta_{\text {emitted }}$ is the value of $\zeta$ at the location where the massless particle was emitted, when the mode $\zeta_{k_{L}}$ was outside the horizon (so that we can neglect the "doppler term" ${ }^{3}$ ). Since $\zeta$ is time independent, $\zeta_{\text {emitted }}=\zeta\left(\vec{x}_{e}=\vec{n} \eta_{0}\right)$ where $\eta_{0}$ is the present value of the conformal time and $\vec{n}$ is the direction that the massless particle is coming from. ${ }^{4}$ We then assume that at the emission time the local physics is completely independent of the long scalar mode, which is well outside the horizon. As usual, when we compare the energy coming from different directions $\zeta_{\text {today }}$ cancels out, see figure 2 .

The discussion so far is identical to the discussion of the Sachs Wolfe effect for the CMB. The only difference is that the spectrum of gravitons is not thermal, but it is instead given by a power law distribution $\left(\omega_{0} / \omega\right)^{2(\nu+1+\epsilon)},(2.2)$. Instead of $(\delta T / T)_{S W}=-\zeta / 5$, we simply vary $\delta \omega_{0} / \omega_{0}=-\zeta / 5$. More explicitly, if we look at the spectrum of gravity waves as a function of frequency $\omega$ coming from the direction $\vec{n}$, we obtain

$$
d \rho(\omega, \vec{n})=d \rho(\omega)\left\{1+\frac{2}{5}(\nu+1+\epsilon)\left[\zeta_{\text {today }}-\zeta_{L}\left(\vec{n} \eta_{0}\right)\right]\right\}
$$

were $d \rho(\omega)$ is the isotropic part of the spectrum, given in (2.2). This result can be derived also using cosmological perturbation theory, performing the computation of the three point function as in [18] and following the evolution to the present, the details will be presented separately [19]. Here we have neglected the integrated Sachs Wolfe effect which is due to the cosmological constant. This can be taken into account, as in the case of photons. Since

\footnotetext{
${ }^{3}$ We have also ignored the doppler term today. It is trivial to put it back in.

${ }^{4}$ Note that $\vec{x}_{e}=\vec{n}\left(\eta_{0}-\eta_{e}\right) \sim \vec{n} \eta_{0}$, and $\vec{x}_{\text {today, here }}=\overrightarrow{0}$.
} 
the same effect is giving rise to fluctuations in the $\mathrm{CMB}$ and in the gravity wave spectrum, we can also write the formula as

$$
d \rho(\omega, \vec{n})=d \rho(\omega)\left\{1-\left(\frac{\delta T}{T}\right)_{S W+I S W} \times k \partial_{k} \log \left\langle N_{k}\right\rangle\right\}
$$

where the subindex indicates the contribution from the Sachs-Wolfe and the integrated Sachs Wolfe effect. The discussion so far has not included the damping effect of the neutrinos [20]. This is expected to be a local effect which will not depend on the long mode. For that reason the final formula as written in (3.3) would also be correct if one inserts the full $\left\langle N_{k}\right\rangle$ expression that includes the effects of the neutrinos. We can view this equation as a consistency condition for a single field inflation. Usually, the consistency condition is discussed for the wavefunction of the universe outside the horizon [18, 21] (see also [22]), but as emphasized in [23] the physical content of that condition is that a local observer cannot notice the long fluctuation. It is a manifestation of the equivalence principle. Furthermore, the final answer could be viewed as arising from a projection effect due to the propagation of the massless particles through the perturbed universe. Furthermore, since the whole effect comes from late time projection effects, the final formula (3.3) is valid also for gravity waves that are generated by any process that happened before the long mode crossed the horizon, such as phase transitions in the early universe, for example. ${ }^{5}$

Any deviation away from this expression (3.3) would be evidence of a second field which would be affecting the relative densities of gravity waves relative to everything else. We think that this would occur in the curvaton models [24-26].

Since the fluctuations are very small, of order $10^{-5}$, these are rather difficult to measure. In addition, we should remember that we are dealing with a stochastic background, so that the observed gravity wave over a small number of cycles is fairly random, and the statement in (3.3) is about the deviation in the variance of that random variable. This means that we need to measure this random variable many times. For the case of random waves, we can view each cycle of the wave as one instance of the random distribution. This means that to reach this accuracy we need to observe of order $10^{10}$ cycles in each angular direction. For waves a frequency of $f[\mathrm{~Hz}]$ we need about $300 / f$ years. ${ }^{6}$

We would like to thank our referee who pointed our attention to the fact that anisotropies also could be present due to individual sources [27, 28] and it might be possible to discriminate them since perturbation that are discussed in the present paper are frequency independent.

\section{Acknowledgments}

We thank V. Mukhanov and specially M. Zaldarriaga for discussions.

The work of VA was supported by the National Science Foundation under Grant No. PHY-1314198. The work of JM was supported in part by U.S. Department of Energy grant de-sc0009988.

\footnotetext{
${ }^{5}$ We thank M. Zadarriaga for making this observation.

${ }^{6}$ This estimate has been made under the assumption that detector has enough sensitivity to measure gravitational wave for each frequency bin.
} 
Open Access. This article is distributed under the terms of the Creative Commons Attribution License (CC-BY 4.0), which permits any use, distribution and reproduction in any medium, provided the original author(s) and source are credited.

\section{References}

[1] A.A. Starobinsky, Spectrum of relict gravitational radiation and the early state of the universe, JETP Lett. 30 (1979) 682 [Pisma Zh. Eksp. Teor. Fiz. 30 (1979) 719] [INSPIRE].

[2] A.A. Starobinsky, A new type of isotropic cosmological models without singularity, Phys. Lett. B 91 (1980) 99 [INSPIRE].

[3] A.H. Guth, The inflationary universe: a possible solution to the horizon and flatness problems, Phys. Rev. D 23 (1981) 347 [inSPIRE].

[4] A.D. Linde, A new inflationary universe scenario: a possible solution of the horizon, flatness, homogeneity, isotropy and primordial monopole problems, Phys. Lett. B 108 (1982) 389 [INSPIRE].

[5] A. Albrecht and P.J. Steinhardt, Cosmology for grand unified theories with radiatively induced symmetry breaking, Phys. Rev. Lett. 48 (1982) 1220 [INSPIRE].

[6] V.F. Mukhanov and G.V. Chibisov, Quantum fluctuation and nonsingular universe (in Russian), JETP Lett. 33 (1981) 532 [Pisma Zh. Eksp. Teor. Fiz. 33 (1981) 549] [inSPIRE].

[7] S.W. Hawking, The development of irregularities in a single bubble inflationary universe, Phys. Lett. B 115 (1982) 295 [INSPIRE].

[8] A.H. Guth and S.Y. Pi, Fluctuations in the new inflationary universe, Phys. Rev. Lett. 49 (1982) 1110 [INSPIRE].

[9] A.A. Starobinsky, Dynamics of phase transition in the new inflationary universe scenario and generation of perturbations, Phys. Lett. B 117 (1982) 175 [INSPIRE].

[10] J.M. Bardeen, P.J. Steinhardt and M.S. Turner, Spontaneous creation of almost scale-free density perturbations in an inflationary universe, Phys. Rev. D 28 (1983) 679 [INSPIRE].

[11] R.K. Sachs and A.M. Wolfe, Perturbations of a cosmological model and angular variations of the microwave background, Astrophys. J. 147 (1967) 73 [Gen. Rel. Grav. 39 (2007) 1929] [INSPIRE].

[12] L.A. Boyle and P.J. Steinhardt, Probing the early universe with inflationary gravitational waves, Phys. Rev. D 77 (2008) 063504 [astro-ph/0512014] [INSPIRE].

[13] K. Nakayama, S. Saito, Y. Suwa and J. Yokoyama, Probing reheating temperature of the universe with gravitational wave background, JCAP 06 (2008) 020 [arXiv:0804.1827] [INSPIRE].

[14] Y. Zhang, X.Z. Er, T.Y. Xia, W. Zhao and H.X. Miao, Exact analytic spectrum of relic gravitational waves in accelerating universe, Class. Quant. Grav. 23 (2006) 3783 [astro-ph/0604456] [INSPIRE].

[15] S. Kuroyanagi, K. Nakayama and S. Saito, Prospects for determination of thermal history after inflation with future gravitational wave detectors, Phys. Rev. D 84 (2011) 123513 [arXiv: 1110.4169] [INSPIRE].

[16] R. Jinno, T. Moroi and K. Nakayama, Inflationary gravitational waves and the evolution of the early universe, JCAP 01 (2014) 040 [arXiv:1307.3010] [INSPIRE]. 
[17] L. Boubekeur, P. Creminelli, J. Norena and F. Vernizzi, Action approach to cosmological perturbations: the $2^{\text {nd }}$ order metric in matter dominance, JCAP 08 (2008) 028 [arXiv:0806.1016] [INSPIRE].

[18] J.M. Maldacena, Non-Gaussian features of primordial fluctuations in single field inflationary models, JHEP 05 (2003) 013 [astro-ph/0210603] [INSPIRE].

[19] V. Alba, to appear (2015).

[20] S. Weinberg, Damping of tensor modes in cosmology, Phys. Rev. D 69 (2004) 023503 [astro-ph/0306304] [INSPIRE].

[21] P. Creminelli and M. Zaldarriaga, Single field consistency relation for the 3-point function, JCAP 10 (2004) 006 [astro-ph/0407059] [INSPIRE].

[22] P. Creminelli, C. Pitrou and F. Vernizzi, The CMB bispectrum in the squeezed limit, JCAP 11 (2011) 025 [arXiv: 1109.1822] [INSPIRE].

[23] E. Pajer, F. Schmidt and M. Zaldarriaga, The observed squeezed limit of cosmological three-point functions, Phys. Rev. D 88 (2013) 083502 [arXiv: 1305. 0824] [InSPIRE].

[24] K. Enqvist and M.S. Sloth, Adiabatic CMB perturbations in pre-big bang string cosmology, Nucl. Phys. B 626 (2002) 395 [hep-ph/0109214] [INSPIRE].

[25] D.H. Lyth and D. Wands, Generating the curvature perturbation without an inflaton, Phys. Lett. B 524 (2002) 5 [hep-ph/0110002] [INSPIRE].

[26] T. Moroi and T. Takahashi, Effects of cosmological moduli fields on cosmic microwave background, Phys. Lett. B 522 (2001) 215 [Erratum ibid. B 539 (2002) 303] [hep-ph/0110096] [INSPIRE].

[27] G. Giampieri and A.G. Polnarev, Detecting an anisotropic gravitational wave background with LISA, Class. Quant. Grav. 14 (1997) 1521 [InSPIRE].

[28] B. Allen and A.C. Ottewill, Detection of anisotropies in the gravitational wave stochastic background, Phys. Rev. D 56 (1997) 545 [gr-qc/9607068] [INSPIRE]. 